\title{
PREDICTING THE ENERGETICS OF HYDROGEN INTERCALATION in Metal Oxides Using Acid-Base Properties
}

SUPPORTING INFORMATION

Evan V. Miu

Department of Chemical and Petroleum Engineering University of Pittsburgh

Pittsburgh, PA 15261
Giannis Mpourmpakis

Department of Chemical and Petroleum Engineering University of Pittsburgh

Pittsburgh, PA 15261

gmpourmp@pitt.edu

James R. McKone

Department of Chemical and Petroleum Engineering

University of Pittsburgh

Pittsburgh, PA 15261

jmckone@pitt.edu 


\section{Tungsten trioxide lattice parameters}

Table S1 compares the experimental and DFT relaxed lattice parameters for monoclinic $\mathrm{WO}_{3}$. Experimental values were taken from [1].

\begin{tabular}{||c|c|c||}
\hline Parameter & Experimental & DFT \\
\hline \hline$a$ & $7.301 \AA$ & $7.453 \AA$ \\
$b$ & $7.539 \AA$ & $7.650 \AA$ \\
$c$ & $7.690 \AA$ & $7.933 \AA$ \\
$\beta$ & $90.892^{\circ}$ & $90.233^{\circ}$ \\
\hline
\end{tabular}

Table S1: Comparison of experimental and DFT relaxed bulk $\mathrm{WO}_{3}$ lattice parameters.

\section{Performance comparison for different tungsten trioxide morphologies}

All experiments reported in the main text were performed on nanoparticulate $\mathrm{WO}_{3}$ produced via ion exchange. This material was selected for its ease of synthesis and its amenability to electrochemical analysis. As a comparison, we also prepared dense films of $\mathrm{WO}_{3}$ by thermally oxidizing sheets of tungsten metal. Briefly, W metal sheets (MTI Corporation MF-W200L) were cut to size and placed in a box furnace for $1 \mathrm{hr}$ at $500{ }^{\circ} \mathrm{C}$ under ambient air.

For this comparison, cyclic voltammetry was performed in a different setup than described in the main text. Since the dense film material was cut from a tungsten foil, this sample could not be supported on a glassy carbon electrode. It was instead tested as a self-supported working electrode using a custom acrylic sandwich-type cell. The working area of this cell setup was $0.495 \mathrm{~cm}^{2}$. Counter and reference electrodes were as described in the main text, as was the potentiostat.

To maintain a good comparison, the nanoparticle material was also tested in this setup. Instead of a glassy carbon substrate, the nanoparticulate $\mathrm{WO}_{3}$ was supported on thin film of Au that was deposited onto standard microscope glass slide using a Plassys MEB550S E-Beam Evaporator. The Au layer was $100 \mathrm{~nm}$ in thickness and adhered to the microscope glass using a $5 \mathrm{~nm}$ Ti film. Nanoparticulate $\mathrm{WO}_{3}$ was doctor-bladed onto this substrate and the full working electrode (microscope glass, $\mathrm{Ti}, \mathrm{Au}, \mathrm{WO}_{3}$ ) was calcined for $1 \mathrm{hr}$ at $500{ }^{\circ} \mathrm{C}$. Figure $\mathrm{S} 1$ shows the results of cyclic voltammetry experiments run at $100 \mathrm{mV} / \mathrm{s}$ in $0.5 \mathrm{M} \mathrm{H}_{2} \mathrm{SO}_{4}$. As can be seen, the $\mathrm{WO}_{3}$ nanoparticles show generally larger current densities along with multiple distinguishable reduction events. The Au background signal is shown to illustrate that the cathodic currents are due to the nanoparticle $\mathrm{WO}_{3}$ and that there is minimal contribution from the Au support. Based on these results, we chose to focus on the nanoparticulate material.

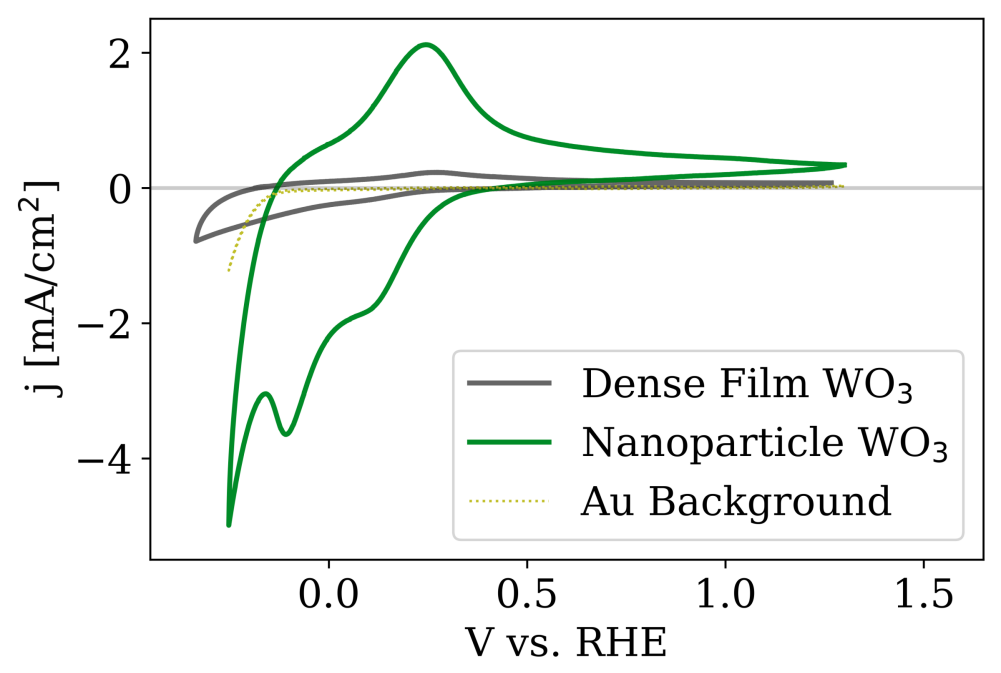

Figure S1: Comparison of $100 \mathrm{mV} / \mathrm{s}$ cyclic voltammetry data on $\mathrm{WO}_{3}$ synthesized via two methods. Nanoparticulate $\mathrm{WO}_{3}$ produced via ion-exchange shows significantly clearer and more reversible redox reactivity than $\mathrm{WO}_{3}$ prepared via thermal oxidation of $\mathrm{W}$ metal. 


\section{Bulk oxide stability and electronic structure heterogeneity}

The heterogeneity of the electronic properties of intercalated oxides can be characterized via the standard deviation, $\sigma$, of a property across the entire unit cell. In the main text, we considered the $\sigma$ of Bader charges on $\mathrm{O}$ and $\mathrm{W}$ atoms as well as $\mathrm{O} p$-band and $\mathrm{W} d$-band centers as potential descriptors for the binding energy model. While these standard deviations were not used in the final model, they are useful quantities that may relate to the stability of the bronzes formed by hydrogen intercalation. Figure S2 shows the relationship between DFT calculated $\mathrm{H}$ binding energy and the standard deviation of $\mathrm{W} d$-band centers.

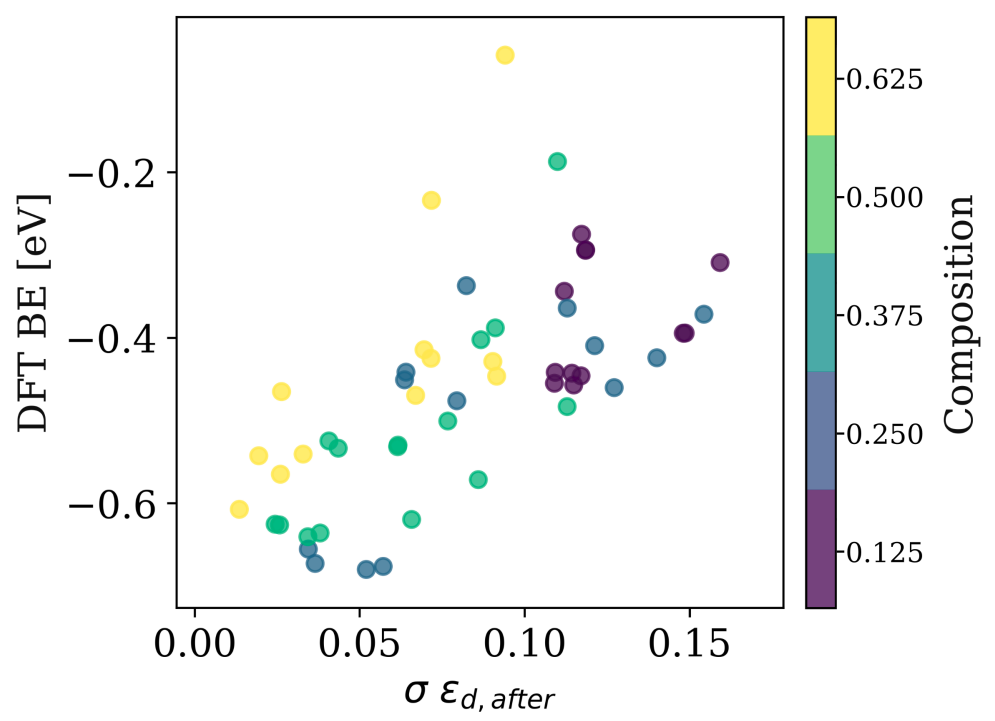

Figure S2: Relationship between DFT calculated binding energy and standard deviation of $\epsilon_{d, \text { after }}$. The coloration follows the composition of the bronze.

The data are partitioned according to composition, as was done for each binding energy model constructed in the main text. Within a constant value of composition (constant color), the oxides with lower values of $\sigma \epsilon_{d, \text { after }}$ generally show more exothermic binding energies. There is a positive linear correlation between the $\sigma \epsilon_{d, a f t e r}$ and binding energy, as revealed in the Pearson correlation matrix shown in Figure 4 of the main text. The standard deviations of Bader charges and band centers were not used in any binding energy model because they are only available after a DFT calculation has already been completed on the associated configuration. 


\section{Decomposition of low-lying unoccupied electronic states}

The total and decomposed electronic densities of states for a $\mathrm{W}$ atom in the $\mathrm{WO}_{3}$ lattice are shown in Figure $\mathrm{S} 3$. The $\mathrm{W}$ $d$-bands dominate the low-lying unoccupied states, consistent with the common description of wide-bandgap transition metal oxides as having metal-centered conduction band edges. As a result, we consider the unoccupied $\mathrm{W} d$-bands as descriptive of acid character.

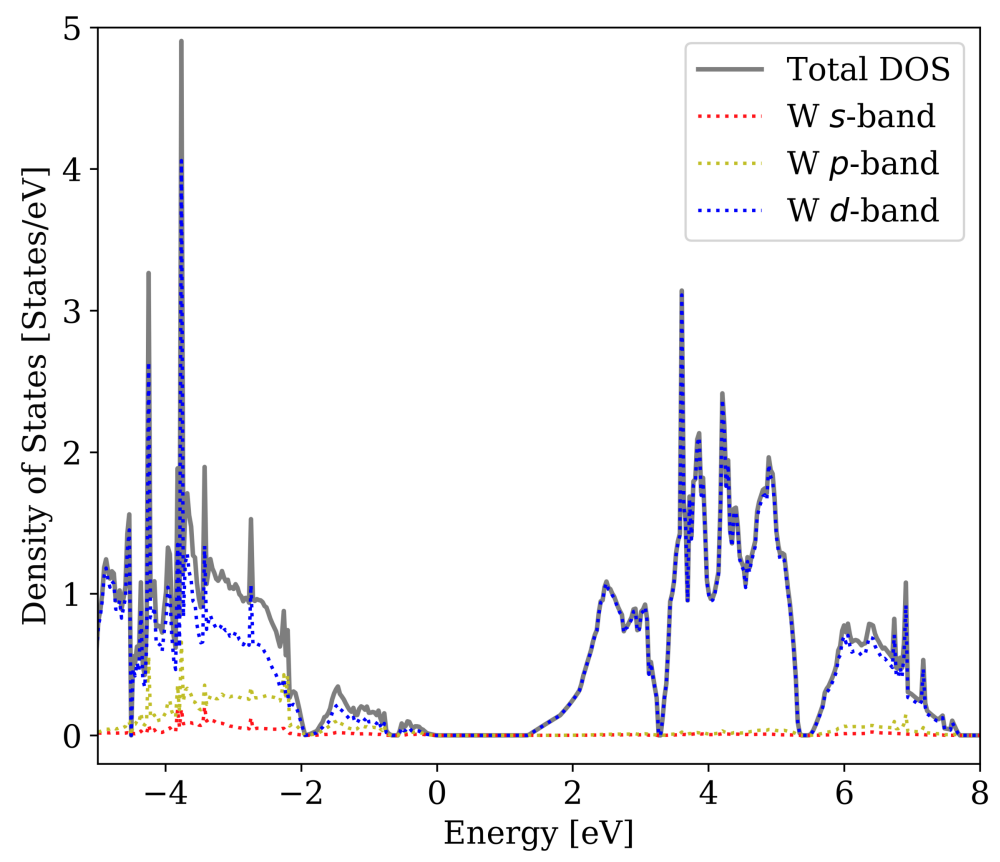

Figure S3: Total and projected electronic density of states on a representative $\mathrm{W}$ atom in a $\mathrm{WO}_{3}$ lattice.

\section{Effect of intercalation on DOS and validation of PBE-D3 electronic structures}

As hydrogen (proton and electron pair) is inserted into the tungsten trioxide lattice, we observe an immediate loss of semiconducting character and a downward shift of the unoccupied $d$-states. Figure $\mathrm{S} 4$ shows this for a representative $\mathrm{W}$ atom (one of the coordinating first neighbors to the $\mathrm{O}$ that receives $\mathrm{H}$ with intercalation) in the $\mathrm{WO}_{3}$ and $\mathrm{H}_{0.125} \mathrm{WO}_{3}$ lattices. Initially, the $\mathrm{WO}_{3}$ in Figure $\mathrm{S} 4 \mathrm{a}$ appears to have a band gap, with the unoccupied $d$-band situated well above the Fermi level. Insertion of the first $\mathrm{H}$ unit drops these unoccupied states down in energy and causes the band to intersect with the Fermi level, suggesting $\mathrm{H}_{0.125} \mathrm{WO}_{3}$ is either a degenerate semiconductor or metallic. This is indicative of the low-lying unoccupied $d$-states in $\mathrm{WO}_{3}$ being filled by the electron introduced via intercalation to $\mathrm{H}_{0.125} \mathrm{WO}_{3}$.

Furthermore, it is well known that PBE (and more generally, the GGA class of functionals) severely underestimates electronic bandgaps. ${ }^{[2]}$ As an alternative, hybrid functionals, such as HSE06, are much better at band gap identifications, with an associated increase in cost. ${ }^{[3]}$ Therefore, to assess whether our approach using PBE-D3 was acceptable, we performed HSE06 calculations on the PBE-D3 optimized geometries to confirm the general trends in expected electronic structures. Our calculations show the $\mathrm{WO}_{3}$ band gap is $1.39 \mathrm{eV}$ by PBE-D3 and 2.49 eV by HSE06. UV-vis measurements shown below indicate an optical gap of $\sim 2.69 \mathrm{eV}$, which agrees better with the HSE06 result than the PBE-D3 one. However, as can be seen in Figure S4, the differences between PBE-D3 and HSE06 DOS only manifest in a constant offset in energies; the overall shape and character of the bands is similar. Therefore, using either of these two functionals would be expected to give similar accuracy in the predicted energetics for H-uptake because the regression models in this work employ free intercepts. This is significant because it is only the slope of the relationships between the descriptors (band centers) and targets (binding energies) that are mathematically important, not necessarily the absolute value of the descriptors themselves. The unoccupied band centers in the intercalated bronze (Figure S4b) are also in near exact agreement between PBE-D3 and HSE06. Although the difference in $\mathrm{WO}_{3}$ unoccupied $d$-band centers is not negligible, the regression models would effectively erase this offset via the intercept. Thus, we conclude that PBE-D3 offers an excellent compromise between accuracy and cost. 

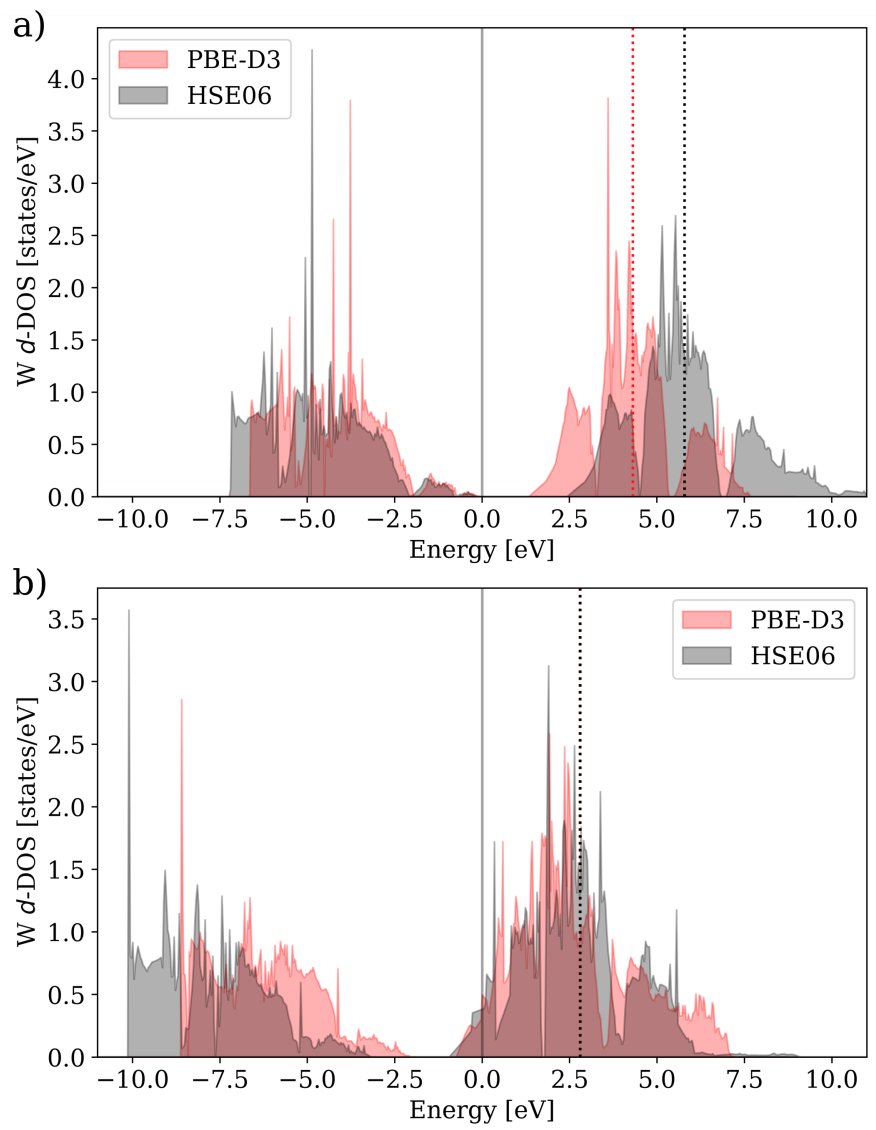

Figure S4: $\mathrm{W} d$-projected DOS for a) $\mathrm{WO}_{3}$ and b) $\mathrm{H}_{0.125} \mathrm{WO}_{3}$ at PBE-D3 and HSE06 levels of theory.

\section{Local vs. global screening model construction}

The initial stage of the computational methodology proposed in the main text comprises a series of local (i.e., binned by hydrogen stoichiometry) linear models. Logistically, these are more useful than one large global model because they enable a step-wise identification of exothermic sites. A global model would require a recursive approach, whereas the step-wise local models efficiently locate hydrogen binding sites, allowing for efficient identification of low-energy bronze configurations.

Figure S5 further motivates the construction of local linear regression models rather than a global one. A clear reversal paradox is observed for the relationship between hydrogen BE and absolute energy. These quantities have a known mathematical relationship (Eq. 1 in the main text). This is represented by the colored lines drawn through each stoichiometry in Figure S5. The black dotted line shows the best fit to the global dataset: the slope of the relationship is inverted and the accuracy is well below that of the local models, demonstrating the utility of considering local linear screening relationships.

\section{Hyperparameter optimization for acid-base kernel ridge regression}

The kernel ridge regression model implemented in the scikit-learn package can be controlled using two hyperparameters. The first is regularization strength, which affects the degree of $L_{2}$ regularization, and the second is the width of the kernel. A Laplacian kernel was selected because it gave the lowest MAE values out of the kernels that were tested. Figure S6 illustrates the average model MAE for training and validation sets of data as functions of the hyperparameters. The combination of parameters that gave the lowest average MAE from cross-validation runs was a regularization strength of 0.03 and a kernel width of 2 . The descriptors were not standardized or normalized because they were all within the same scale. 


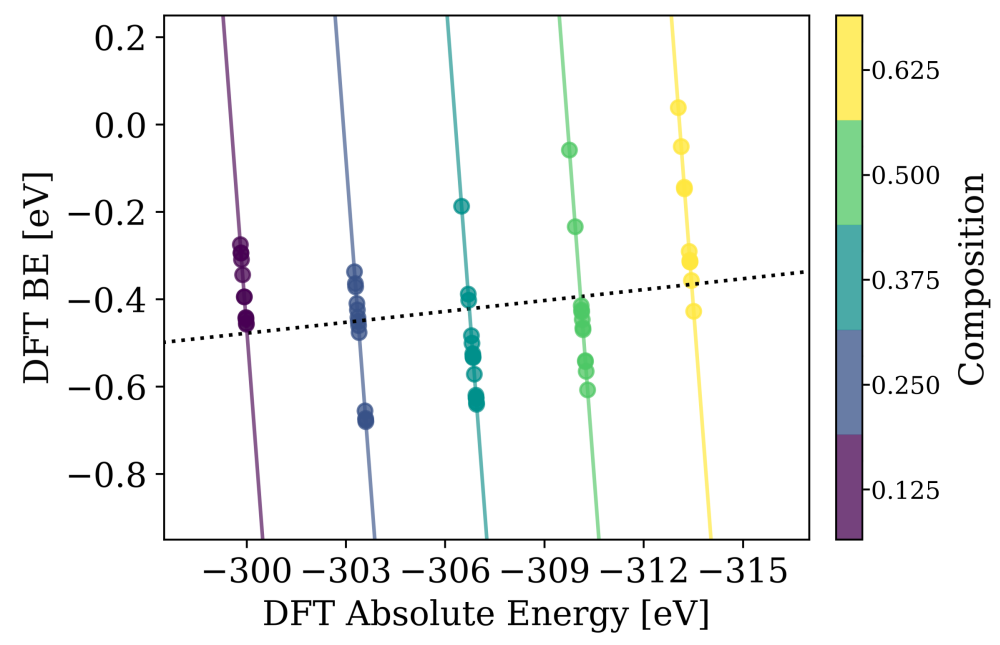

Figure S5: Comparison of absolute DFT energy to hydrogen binding energy, split by composition.

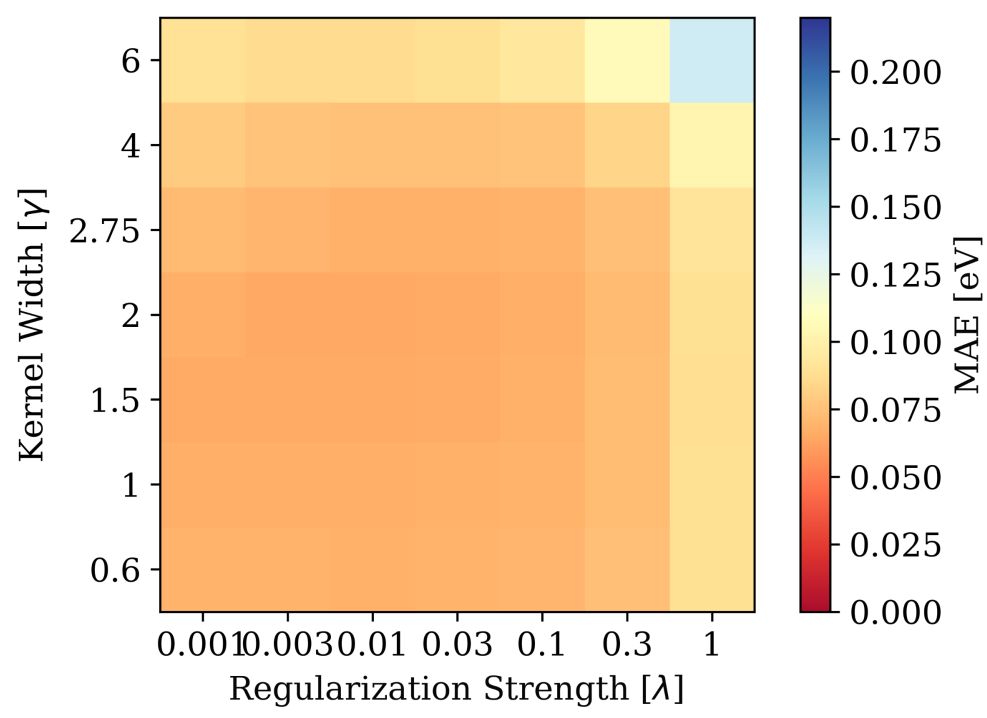

Figure S6: Average MAE of acid-base models generated by 10-fold cross-validation repeated 200 times, shown as a function of regularization strength and kernel smearing width.

\section{UV-vis measurements as a function of bronze composition}

Intercalation of tungsten trioxide is known to induce electrochromism, characterized by a shift from bright yellow-green to dark blue-indigo. ${ }^{[4]}$ We performed UV-vis measurements in reflectance mode to investigate the relationship between polarization, optical response, and semiconducting character of the intercalated bronzes. The samples were polarized for 5 minutes at potentials of $+0.45,+0.15$, and $0.00 \mathrm{~V}$ vs. RHE, corresponding to voltammogram features that we have assigned to the parent oxide, formation of $\mathrm{H}_{0.250} \mathrm{WO}_{3}$, and formation of $\mathrm{H}_{0.500} \mathrm{WO}_{3}$, respectively. The cell setup for pretreatment was identical to the CV and EIS setups. Immediately after pretreatment was finished, the films were lifted from the glassy carbon electrode using adhesive tape (Scotch magic tape) and then pressed onto a glass microscope slide. This was done to minimize contact with air, which re-oxidizes the bronze to $\mathrm{WO}_{3}$. Tests showed that samples collected and preserved in this manner retained their coloration for several days after preparation.

A Craic QDI 2010 UV-vis/NIR microspectrophotomer in reflectance mode was used to collect the spectra shown in Figure S7. $\mathrm{KCl}$ was used as a $100 \%$ reflectance reference, and spectra were collected in the $300-800 \mathrm{~nm}$ range. Reflectance data were transformed to the corresponding Kubelka-Munk function, $F(R)=(1-R)^{2} /(2 R)$, where $R=$ 
the percent reflectance. Tauc plots were then constructed considering $\mathrm{WO}_{3}$ as an indirect semiconductor. Figure $\mathrm{S} 7 \mathrm{a}$ illustrates $F(R)$ versus wavelength for three levels of intercalation. $\mathrm{H}_{0.250} \mathrm{WO}_{3}$ shows an marked increase in absorption across the visible spectrum relative to $\mathrm{WO}_{3}$. Intercalation to $\mathrm{H}_{0.500} \mathrm{WO}_{3}$ shows an even larger absorbance across the visible wavelengths, illustrating composition-dependent coloration as expected for an electrochromic oxide.

The Tauc interpretation of the reflectance data for $\mathrm{WO}_{3}$, as seen in Figure $\mathrm{S} 7 \mathrm{~b}$, shows clear semiconducting character, with a linear region fit returning an indirect band gap of $2.69 \mathrm{eV}$. The hydrogen bronzes, by contrast, show no evidence for a bandgap. This is consistent with the impedance data in the following section, where intercalation brings about a drastic reduction in charge transfer resistance. Altogether, this suggests a transition from semiconducting to metallic behavior that occurs with intercalation, as expected from prior work on $\mathrm{H}_{\mathrm{x}} \mathrm{WO}_{3}{ }^{[5,6]}$ and the DOS data shown above. Furthermore, the semiconducting profile of the Tauc plot and the yellow-green coloration of $\mathrm{WO}_{3}$ suggest that the starting material is not significantly substoichiometric. $\mathrm{WO}_{3-\mathrm{x}}$ is known to have increased absorption across visible wavelengths and appears dark blue even at very small values of $x .^{[7]}$
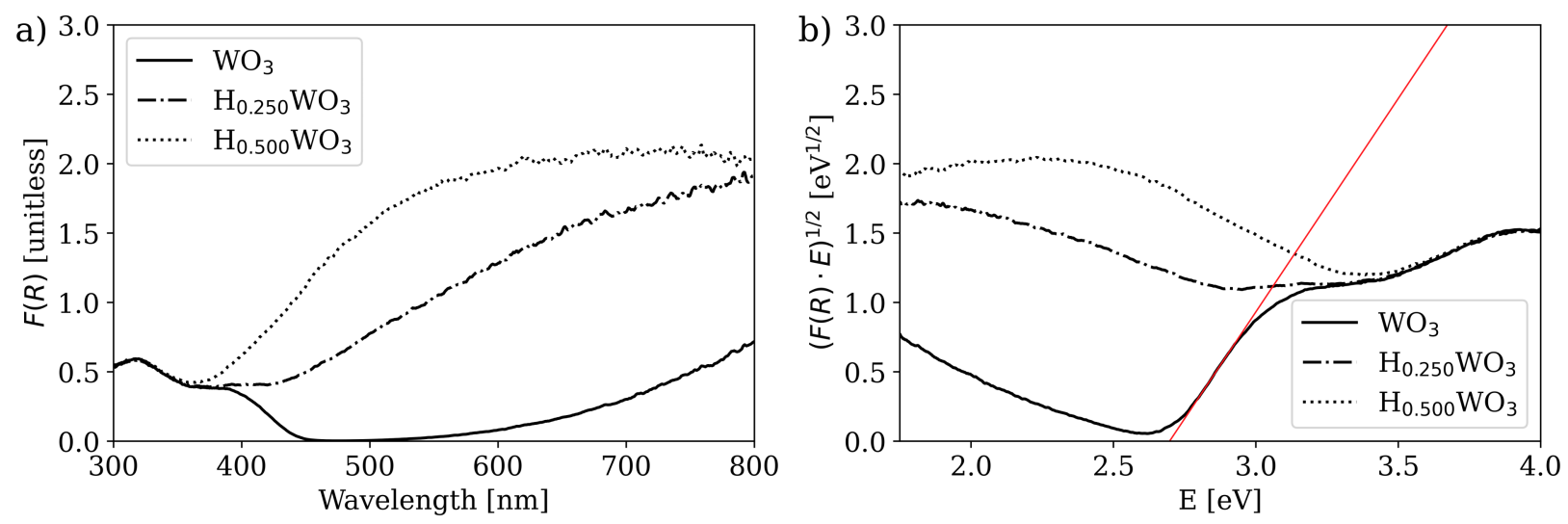

Figure S7: a) Kubelka-Munk function, $F(R)$, and b) Tauc plots for three levels of hydrogen intercalation in $\mathrm{WO}_{3}$. The stiochimetries are labeled according to the assignments from modeling and voltammetric features in the main text. The red line in b) corresponds to the fit of the linear Tauc region, which returns an indirect band gap of $2.69 \mathrm{eV}$.

\section{Structural evolution with bronze composition and the validity of a fixed-volume calculation approach}

Prior work has shown that $\mathrm{H}$-intercalation in the $\mathrm{H}_{\mathrm{x}} \mathrm{WO}_{3}$ bronze system coincides with an increase in crystal symmetry. ${ }^{[8]}$ Initially, the low symmetry monoclinic material shows three lattice parameters that are distinct. From $\mathrm{H}_{0.150} \mathrm{WO}_{3}$ to $\mathrm{H}_{0.500} \mathrm{WO}_{3}$ there is an expected transition through two tetragonal crystal structures. Accordingly, we performed x-ray diffraction (XRD) measurements to track this structural change in our material and provide further evidence of hydrogen intercalation. Measurements were taken with a Bruker D8 Discover instrument equipped with a LynxEye detector using $\mathrm{Cu} \mathrm{K}-\alpha$ radiation and step increments of $0.01^{\circ}$ at $1.5 \mathrm{secs} / \mathrm{step}$. Figure S8 shows the results of these measurements on samples prepared identically to those described above for UV-vis experiments.

The XRD pattern for the initial material is consistent with monolinic $\mathrm{WO}_{3}$ exhibiting a high degree of crystallographic orientation (texturing), where increased intensity in the (002) feature occurs due to the disk-like shape of the nanoparticles. After H-intercalation at $+0.15 \mathrm{~V}$ vs. RHE, which we expect to coincide with the formation of $\mathrm{H}_{0.250} \mathrm{WO}_{3}$, we observe a slight expansion in d-spacing (decrease in $2 \theta$ ) for the (002) reflection along with the disappearance of the shoulder associated with the (020) crystal direction. This suggests the (002) and (020) directions adopt similar $\mathrm{d}$-spacings, reflecting an increase in symmetry. By contrast, further polarization to $0.00 \mathrm{~V}$ vs. RHE shows a decrease in d-spacing for the main feature and a coalescence of the diffraction features, implying a further increase in crystal symmetry.

The computational framework proposed by this work was accelerated by restricting the DFT calculations to fixed volume and constant cell parameters for each intercalation. This was done to reduce the computational cost of geometric optimizations, where volume and cell parameter relaxations introduce more degrees of freedom into the overall structure search. It is possible, however, that this simplification introduced errors by ignoring the energy change associated with changes in the cell shape induced by intercalation. Furthermore, the fixed volume approach restricts the structure to a single system (monoclinic, in this case). To assess the accuracy of the energies obtained by fixed volume approach, we 


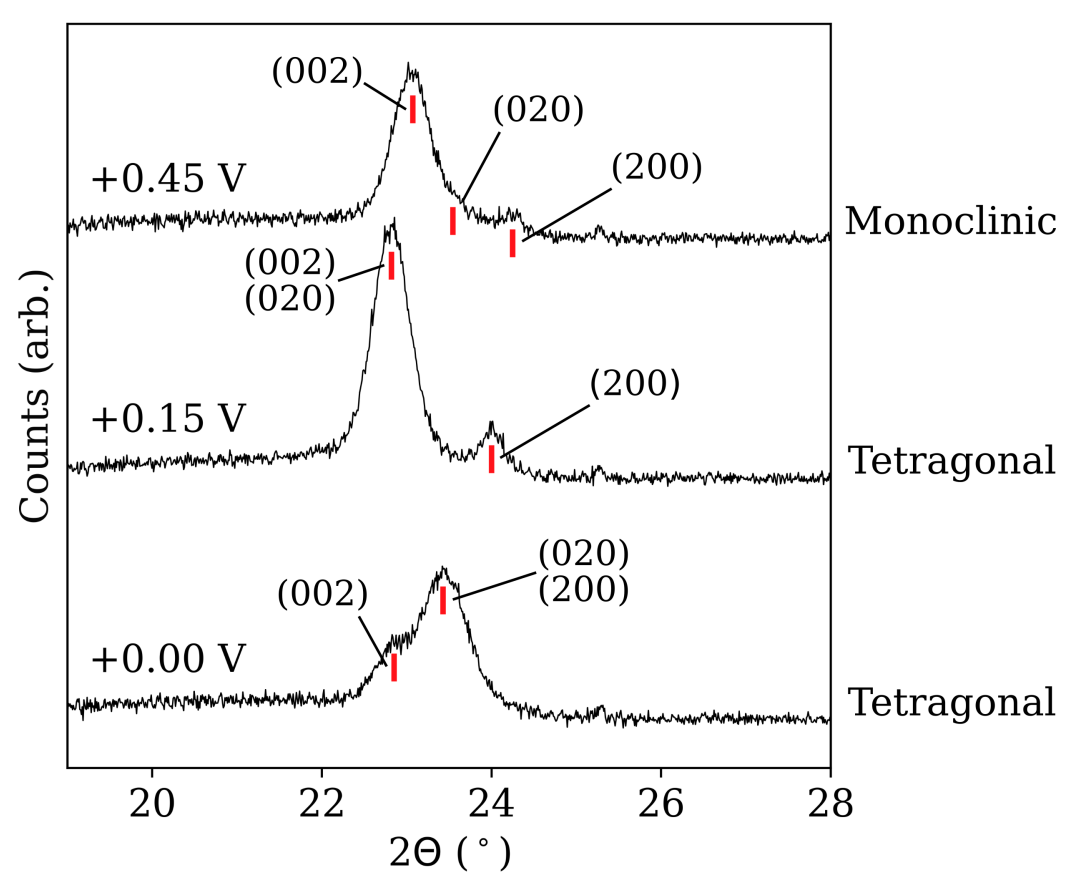

Figure S8: X-ray diffractograms for various levels of hydrogen intercalation, shown as polarization vs. RHE, illustrating a clear increase in crystal symmetry.

performed full volume and cell parameter relaxations of the most exothermic configurations for each investigated bronze stoichiometry. The results are listed in Table S2. Volume relaxation energies were calculated as the ground-state energy of the shape- and volume-relaxed structure minus the fixed volume structure energy (those reported as the convex hull in the main text). Intercalation potential corrections follow the calculation in the main text, where we calculated the slope of the convex hull between the stoichiometries of interest. The potential corrections due to volume relaxation resulted in a systematic positive shift in the predicted intercalation potentials, but the differences were similar in magnitude to the $95 \%$ confidence intervals given by our Boltzmann weighting procedure. The exception is $\mathrm{H}_{0.125} \mathrm{WO}_{3}$, which shifted positive by $>0.1 \mathrm{~V}$. While this does not change the main conclusions from the fixed-volume calculations, it adds further evidence that either $\mathrm{H}_{0.125} \mathrm{O}_{3}$ does not form or the DFT-predicted equilibrium potential for this intercalation event is less accurate than for higher $\mathrm{H}$-stoichiometries.

\begin{tabular}{|c|c|c|c|}
\hline Stoichiometry & Volume Relaxation Energy [eV] & Change in Potential [eV] & $95 \%$ Confidence Interval $[\mathrm{eV}]$ \\
\hline $\mathrm{WO}_{3}$ & - & - & - \\
\hline $\mathrm{H}_{0.125} \mathrm{WO}_{3}$ & -0.1138 & +0.1138 & 0.0350 \\
\hline $\mathrm{H}_{0.250} \mathrm{WO}_{3}$ & -0.1409 & +0.0271 & 0.0205 \\
\hline $\mathrm{H}_{0.375} \mathrm{WO}_{3}$ & -0.1639 & +0.0230 & 0.0435 \\
\hline $\mathrm{H}_{0.500} \mathrm{WO}_{3}$ & -0.2095 & +0.0456 & 0.0526 \\
\hline
\end{tabular}

Table S2: Volume relaxation energy corrections and the resulting change in predicted intercalation potentials for each stoichiometry, compared to the $95 \%$ confidence intervals calculated from the fixed volume configuration ensembles.

With the volume and cell shape changes in hand, we can also obtain information about the changing crystal structure with intercalation. Using the powder diffraction pattern module included in VESTA, we identified the (002), (020), and (200) d-spacings that are calculated from the volume relaxed structures. These are listed in Table S3. We observe three distinct spacings for the initial material, followed by an expansion of the (002) spacing in $\mathrm{H}_{0.250} \mathrm{WO}_{3}$ as was observed via XRD. Interestingly, the (200) and (020) spacings were found to be almost the same, rather than the (002) and (020) combination that is expected upon inspection of the XRD data. Despite this disagreement, the grouping of the (200) and (020) spacings determined by DFT highlights the expected increase in symmetry, as well as the slight expansion of the lattice. The $\mathrm{H}_{0.500} \mathrm{WO}_{3}$ spacings follow this trend as well, and show the (200) and (020) spacings are similar, with (002) distinct. This finding is in line with the XRD pattern for $\mathrm{H}_{0.500} \mathrm{WO}_{3}$, showing that the general trend in increased 
symmetry with hydrogen intercalation is captured by the volume-relaxed DFT calculations. In summary, the fixed volume approach seems appropriate for energetic predictions, and additional volume relaxations can be performed for the most exothermic configurations to elucidate additional structural information.

\begin{tabular}{||c|c|c|c||}
\hline Stoichiometry & $(002)$ & $(020)$ & $(200)$ \\
\hline \hline $\mathrm{WO}_{3}$ & 3.966 & 3.825 & 3.727 \\
$\mathrm{H}_{0.250} \mathrm{WO}_{3}$ & 4.036 & 3.771 & 3.706 \\
$\mathrm{H}_{0.500} \mathrm{WO}_{3}$ & 4.033 & 3.739 & 3.765 \\
\hline
\end{tabular}

Table S3: Calculated d-spacings in nm for volume-relaxed cells corresponding to stoichometries investigated via XRD.

\section{Control experiments for window-opening voltammetry}

To rule out the effect of contaminants or exogenous components of the electrochemical cell on the observed oxidation feature at $\sim 1.5 \mathrm{~V}$ vs. RHE, we performed the control experiment shown in Figure S9. The red curve represents the initial, positive-going sweep; this is also indicated by the red arrow. Non-intercalated bronze was polarized until the onset of oxygen evolution, where there was no observation of any anodic current in the strongly oxidizing region. However, cycling to these positive potentials after a negative-going sweep that formed $\mathrm{H}_{\mathrm{x}} \mathrm{WO}_{3}$ resulted in the emergence of peak III alongside a broad anodic current across the positive-going sweep. This is consistent with kinetic or transport-limited oxidation, as discussed in the main text.

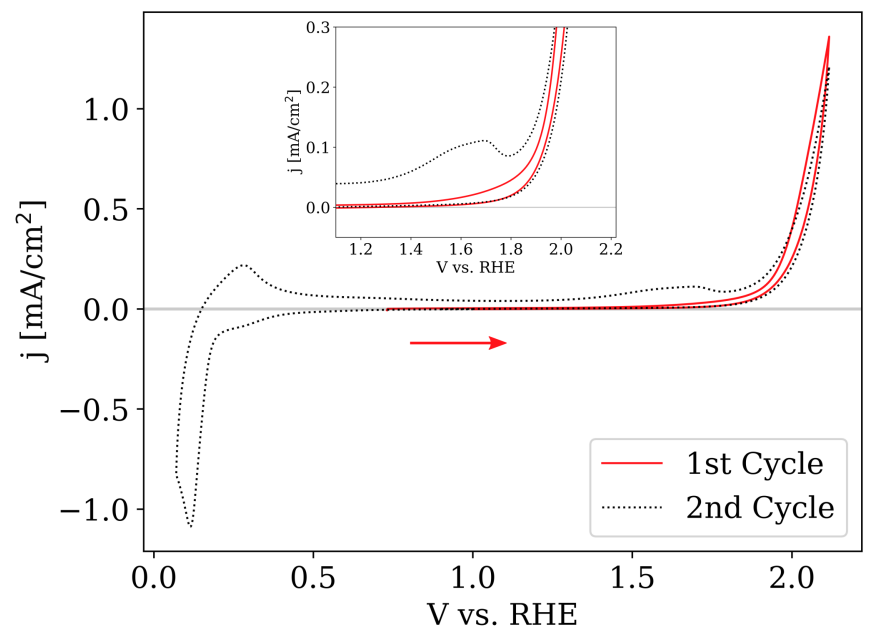

Figure S9: Comparison of two positive-going sweeps at $1 \mathrm{mV} / \mathrm{s}$. The solid red curve shows oxidation of the nonintercalated metal oxide, and the dotted black curve shows oxidation of an intercalated oxide. Inset is an up-close look at the anodic feature at $\sim 1.5 \mathrm{~V}$ vs. RHE.

\section{EIS data fits}

The raw electrochemical impedance spectroscopy (EIS) data and equivalent circuit fits are shown in Figure S10. Raw data are shown as circles, where the calculated fits are shown as solid lines. Fit parameters are shown in the main text in Figure 10.

\section{Scan rate effect on intercalation and de-intercalation}

To use the voltammetry data in Figures 8 and 9 of the main text as indicators of equilibrium potentials, the scan rate must be slow enough to approximate equilibrium and not introduce significant peak shifts associated with kinetic or transport limitations. Figure S11 compares the $1 \mathrm{mV} / \mathrm{s}$ voltammogram shown in Figure 8 of the main text against a slower $0.3 \mathrm{mV} / \mathrm{s}$ scan rate in an identical cell setup. As can be seen, there is a slight positive shift in the first two 

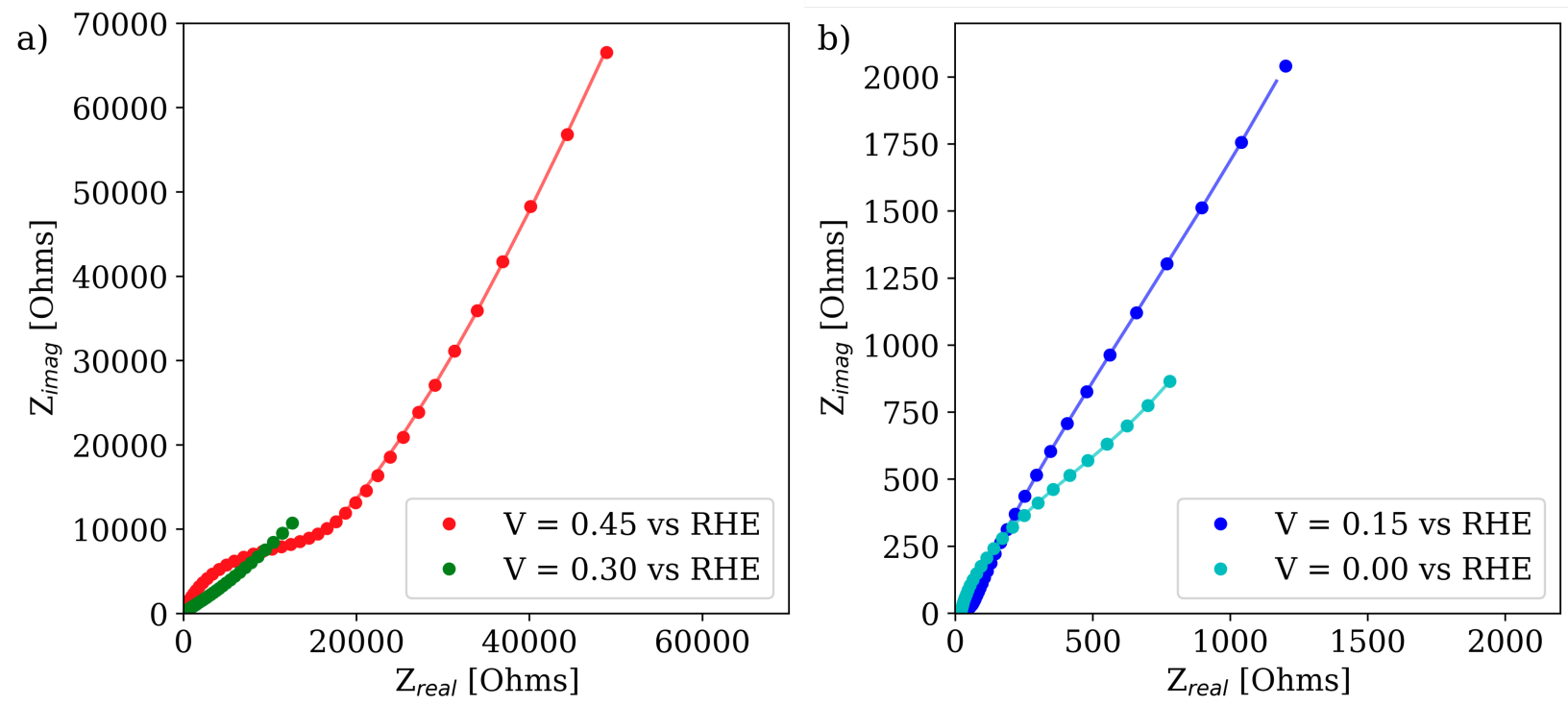

Figure S10: Nyquist plots of the complex plane impedance data for the $\mathrm{H}_{\mathrm{x}} \mathrm{WO}_{3}$ system at varying DC potentials: a) +0.45 and $+0.30 \mathrm{~V}$ vs. RHE; b) +0.15 and $0.00 \mathrm{~V}$ vs. RHE. Note the difference in magnitudes for the axes in a) and b).

reduction features, and a very small negative shift in the corresponding oxidation features. These differences do not significantly influence the identification of equilibrium potentials made using the $1 \mathrm{mV} / \mathrm{s}$ scan rate data.

Interestingly, the third reduction feature associated with the $\mathrm{H}_{0.500} \mathrm{WO}_{3} / \mathrm{H}_{0.625} \mathrm{WO}_{3}$ redox couple is shifted slightly more negative in the slower scan rate data. It is likely that this feature is broad and overlaps with the large shoulder of the second reduction feature. This is motivated by the magnitudes of the charges shown in Figure S11. The total charge associated with reductions are slightly larger than that for oxidation, which is broadly consistent with background hydrogen evolution. It is noteworthy, however, that peak III disappeared with the slower sweep. This is again consistent with the notion of "stranded hydrogen," where the slow scan rate allows the diffusion of bulk hydrogen equivalents to keep up with the changing electrode potential.

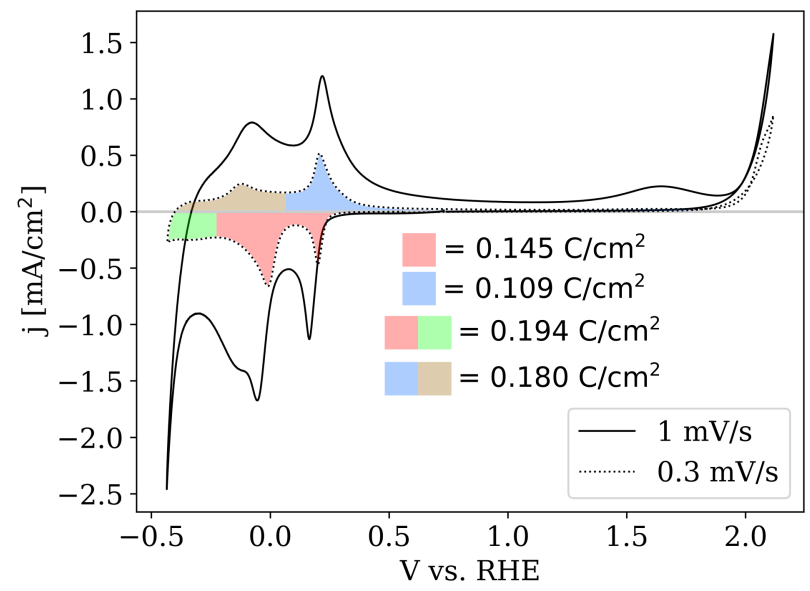

Figure S11: Comparison of $1 \mathrm{mV} / \mathrm{s}$ and $0.3 \mathrm{mV} / \mathrm{s}$ scan rates and the resulting shifts in voltammograms.

\section{Anion effects on hydrogen intercalation}

We also tested the effect of a different supporting electrolyte on the cyclic voltammograms, to determine whether equilibrium potentials were signifcantly influenced by anion effects. Figure S12 shows the intercalation and oxidation of $\mathrm{H}_{\mathrm{x}} \mathrm{WO}_{3}$ in sulfuric and perchloric acids. The use of perchloric acid results in a slight negative shift in the reductive 
peaks by up to $\sim 60 \mathrm{mV}$. There is no concurrent shift in the oxidative peaks. This difference may be indicative of influence by anion adsorption (e.g., displacement of bisulfate concurrent with H-intercalation), but it again has no significant impact on the assignment of redox features with $\mathrm{H}$-stoichiometries and again remains within the error of the computational predictions (which did not consider anionic adsorbates).

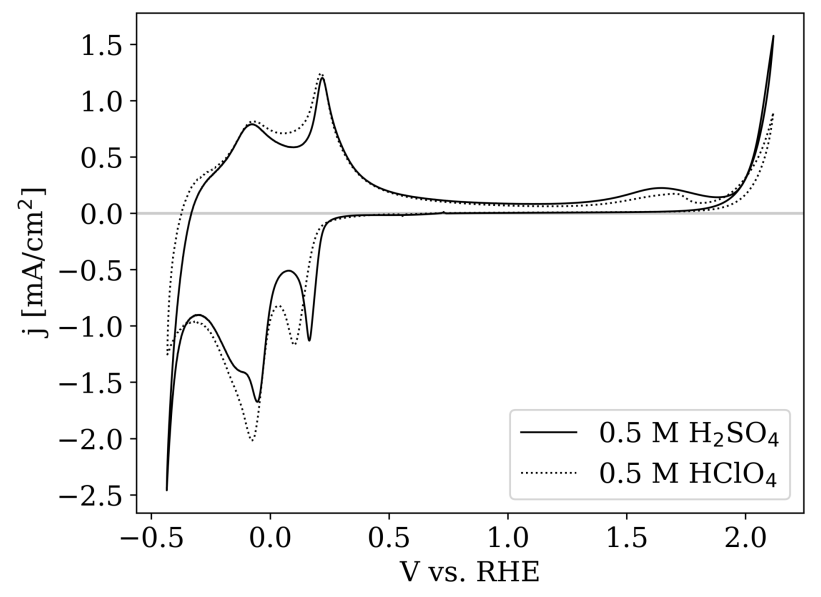

Figure S12: Comparison of voltammograms in $0.5 \mathrm{M} \mathrm{H}_{2} \mathrm{SO}_{4}$ and $0.5 \mathrm{M} \mathrm{HClO}_{4}$.

\section{Disk-like morphology of synthesized nanoparticles}

We previously reported the synthesis procedure for the nanoparticulate $\mathrm{WO}_{3}$ used here, along with extensive materials characterization. ${ }^{9]}$ To confirm that the specific samples used for this study were comparable to that in our prior report, Figure S13 depicts a representative SEM image. A Zeiss Sigma 500VP field emission scanning electron microscope was used to collect the image using an accelerating voltage of $10 \mathrm{keV}$ and a backscatter detector. The nanoparticles clearly exhibit a disk shape with diameters of a few hundred nanometers, consistent with our prior report. XRD measurements of the $\mathrm{WO}_{3}$ are also consistent with previous measurements.

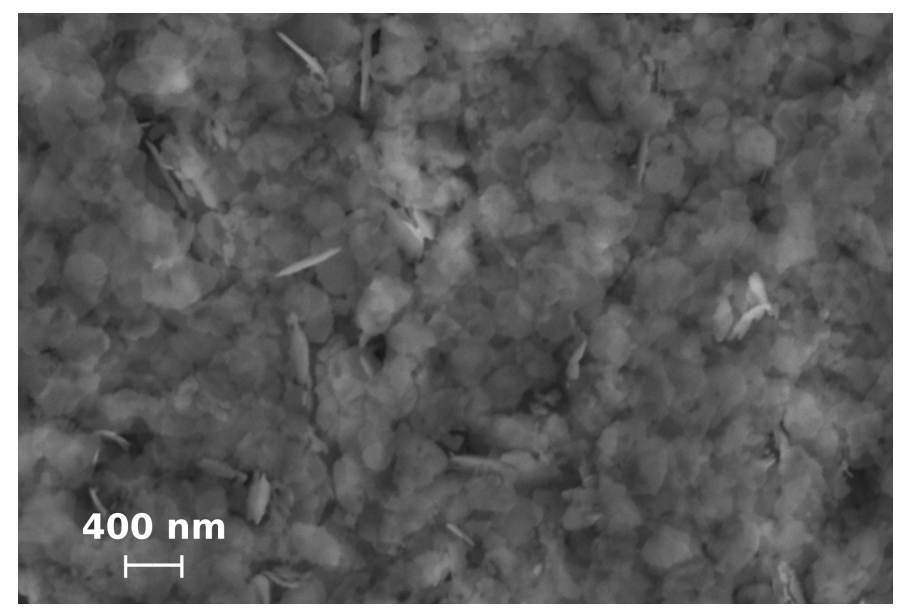

Figure S13: Scanning electron micrograph of a representative sample of as-synthesized nanoparticle $\mathrm{WO}_{3}$. 


\section{References}

[1] Woodward, P.; Sleight, A.; Vogt, T. Structure Refinement of Triclinic Tungsten Trioxide. Journal of Physics and Chemistry of Solids, 1995, 56, 1305-1315.

[2] Marsman, M.; Paier, J.; Stroppa, A.; Kresse, G. Hybrid Functionals Applied to Extended Systems. Journal of Physics: Condensed Matter, 2008, 20(6), 064201-064209.

[3] Wang, F.; Di Valentin, C.; Pacchioni, G. Electronic and Structural Properties of WO3: A Systematic Hybrid DFT Study. The Journal of Physical Chemistry C, 2011, 115(16), 8345-8353.

[4] Glemser, O.; Sauer, H. Die Zusammensetzung von Wolframblau. Zeitschrift für Anorganische Chemie, 1943, 252, $160-163$.

[5] Shanks, H.; Sidles, P.; Danielson, G. Electrical Properties of the Tungsten Bronzes. Advances in Chemistry, 1963, 39, 237-245.

[6] Leng, X.; Pereiro, J.; Strle, J.; Dubuis, G.; Bollinger, A. T.; Gozar, A.; Wu, J.; Litombe, N.; Panagopoulos, C.; Pavuna, D.; Božović, I. Insulator to Metal Transition in $\mathrm{WO}_{3}$ Induced by Electrolyte Gating. npj Quantum Materials, 2017, 2(1), 1-7.

[7] Paik, T.; Cargnello, M.; Gordon, T. R.; Zhang, S.; Yun, H.; Lee, J. D.; Woo, H. Y.; Oh, S. J.; Kagan, C. R.; Fornasiero, P.; Murray, C. B. Photocatalytic Hydrogen Evolution from Substoichiometric Colloidal $\mathrm{WO}_{3-\mathrm{x}} \mathrm{Nanowires}$ ACS Energy Letters, 2018, 3(8), 1904-1910.

[8] Genin, C.; Driouiche, A.; Gerand, B.; Figlarz, M. Hydrogen Bronzes of New Oxides of the $\mathrm{WO}_{3}-\mathrm{MoO}_{3} \mathrm{System}$ with Hexagonal, Pyrochlore and $\mathrm{ReO}_{3}$-type Structures. Solid State Ionics, 1992, 53, 315-323.

[9] Miu, E. V.; McKone, J. R. Comparisons of $\mathrm{WO}_{3}$ Reduction to $\mathrm{H}_{\mathrm{x}} \mathrm{WO}_{3}$ Under Thermochemical and Electrochemical Control. Journal of Materials Chemistry A, 2019, 7, 23756-23761. 